\title{
Bidirectional Relationship between Functional Connectivity and Amyloid- $\beta$ Deposition in Mouse Brain
}

\author{
Adam W. Bero, ${ }^{1,2,6,7 *}$ Adam Q. Bauer, ${ }^{3 \star}$ Floy R. Stewart, ${ }^{1,2,6,7}$ Brian R. White, ${ }^{3,8}$ John R. Cirrito, ${ }^{1,6,7}$ Marcus E. Raichle, ${ }^{1,3,4,5}$ \\ Joseph P. Culver, ${ }^{3,4,8}$ and David M. Holtzman ${ }^{1,2,6,7}$ \\ Departments of ${ }^{1}$ Neurology, ${ }^{2}$ Developmental Biology, ${ }^{3}$ Radiology, ${ }^{4}$ Biomedical Engineering, and ${ }^{5}$ Anatomy and Neurobiology, ${ }^{6}$ Hope Center for \\ Neurological Disorders, and ${ }^{7}$ Charles F. and Joanne Knight Alzheimer's Disease Research Center, Washington University School of Medicine, St. Louis, \\ Missouri 63110, and ${ }^{8}$ Department of Physics, Washington University, St. Louis, Missouri 63130
}

Brain region-specific deposition of extracellular amyloid plaques principally composed of aggregated amyloid- $\beta$ (A $\beta$ ) peptide is a pathological signature of Alzheimer's disease (AD). Recent human neuroimaging data suggest that resting-state functional connectivity strength is reduced in patients with $\mathrm{AD}$, cognitively normal elderly harboring elevated amyloid burden, and in advanced aging. Interestingly, there exists a striking spatial correlation between functional connectivity strength in cognitively normal adults and the location of $\mathrm{A} \beta$ plaque deposition in AD. However, technical limitations have heretofore precluded examination of the relationship between functional connectivity, $\mathrm{A} \beta$ deposition, and normal aging in mouse models. Using a novel functional connectivity optical intrinsic signal (fcOIS) imaging technique, we demonstrate that $\mathrm{A} \beta$ deposition is associated with significantly reduced bilateral functional connectivity in multiple brain regions of older APP/PS1 transgenic mice. The amount of A $\beta$ deposition in each brain region was associated with the degree of local, age-related bilateral functional connectivity decline. Normal aging was associated with reduced bilateral functional connectivity specifically in retrosplenial cortex. Furthermore, we found that the magnitude of regional bilateral functional correlation in young APP/PS1 mice before A $\beta$ plaque formation was proportional to the amount of region-specific plaque deposition seen later in older APP/PS1 mice. Together, these findings suggest that A $\beta$ deposition and normal aging are associated with region-specific disruption of functional connectivity and that the magnitude of local bilateral functional connectivity predicts regional vulnerability to subsequent $\mathrm{A} \beta$ deposition in mouse brain.

\section{Introduction}

Alzheimer's disease $(\mathrm{AD})$ is the most common cause of dementia, characterized by progressive structural and functional deterioration of higher-order neural networks. A histopathological hallmark of $\mathrm{AD}$ is the presence of extracellular plaques primarily composed of aggregated amyloid- $\beta$ (A $\beta$ ) peptide. Normally, A $\beta$ is produced in neurons and present in the extracellular space of the brain in soluble form (Selkoe, 2001). During AD pathogenesis, soluble $\mathrm{A} \beta$ aggregates into oligomers and insoluble extracellular amyloid plaques in a concentration-dependent manner (Yan et al., 2009). In AD, amyloid plaque deposition is most prominent in the default-mode network (DMN), a network of brain areas where metabolism is preferentially elevated during

\footnotetext{
Received Nov. 22, 2011; revised Jan. 14, 2012; accepted Feb. 7, 2012.

Author contributions: A.W.B., A.Q.B., B.R.W., J.R.C., M.E.R., J.P.C., and D.M.H. designed research; A.W.B., A.Q.B., and F.R.S. performed research; J.R.C. and D.M.H. contributed unpublished reagents/analytic tools; A.W.B., A.Q.B., F.R.S., B.R.W., M.E.R., and J.P.C. analyzed data; A.W.B., A.Q.B., J.R.C., M.E.R., J.P.C., and D.M.H. wrote the paper.

This work was supported by NIH Grants AG033452 (to A.W.B.), T90-DA022871 (to B.R.W.), R01-EB008085 and R01-EB009233 (to J.P.C.), the Medical-Scientist Training Program (B.R.W.), Ellison Medical Foundation (D.M.H.), and Cure Alzheimer's Fund (D.M.H.).

${ }^{*}$ A.W.B. and A.Q.B. contributed equally to this work.

The authors declare no competing financial interests.

Correspondence should be addressed to Dr. David M. Holtzman, Andrew B. and Gretchen P. Jones Professor and Chair, Department of Neurology, Washington University School of Medicine, 660 South Euclid Avenue, Campus Box 8111, Saint Louis, M0 63110. E-mail: holtzman@neuro.wustl.edu.

DOI:10.1523/JNEUROSCI.5845-11.2012

Copyright $\odot 2012$ the authors $\quad 0270-6474 / 12 / 324334-07 \$ 15.00 / 0$
}

undirected mentation (Buckner et al., 2005; Vlassenko et al., 2010). Interestingly, functional connectivity MRI data suggest that functional connectivity is disrupted in patients with $\mathrm{AD}$ (Greicius et al., 2004; Jones et al., 2011), cognitively normal elderly with elevated amyloid burden (Hedden et al., 2009; Sheline et al., 2010; Drzezga et al., 2011; Mormino et al., 2011), and in advanced aging (Andrews-Hanna et al., 2007). Moreover, recent data suggest that the relationship between functional connectivity and $\mathrm{A} \beta$ deposition is bidirectional; the DMN exhibits high intrinsic functional connectivity in healthy brain and is preferentially susceptible to $\mathrm{A} \beta$ deposition in AD (Buckner et al., 2009).

In mouse models of $\beta$-amyloidosis, $A \beta$ plaque deposition is associated with myriad neurotoxic effects, including neuritic dystrophy, impaired synaptic integration, and calcium dyshomeostasis (Stern et al., 2004; Kuchibhotla et al., 2008; Meyer-Luehmann et al., 2008). However, because technical limitations have precluded examination of systems-level functional connectivity in model systems, the relationship between intrinsic functional connectivity and $\mathrm{A} \beta$ deposition in mouse brain remains unclear. We designed a novel functional connectivity optical intrinsic signal (fcOIS) imaging approach (White et al., 2011) wherein changes in reflected light intensity from the surface of the brain are converted to changes in local hemoglobin concentration and used to measure intrinsic functional connectivity. Herein, we use fcOIS to determine whether A $\beta$ deposition or normal aging is associated with functional connectivity disruption in APPswe/PS1 $\triangle E 9$ (APP/PS1) transgenic and wild-type 
A

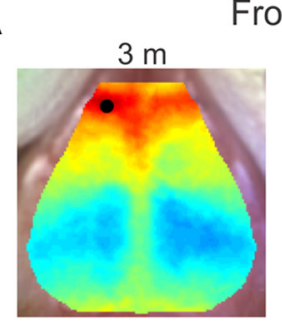

C

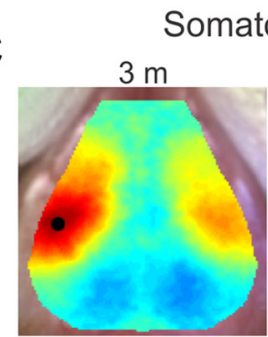

E

E

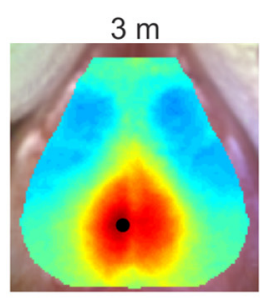

G
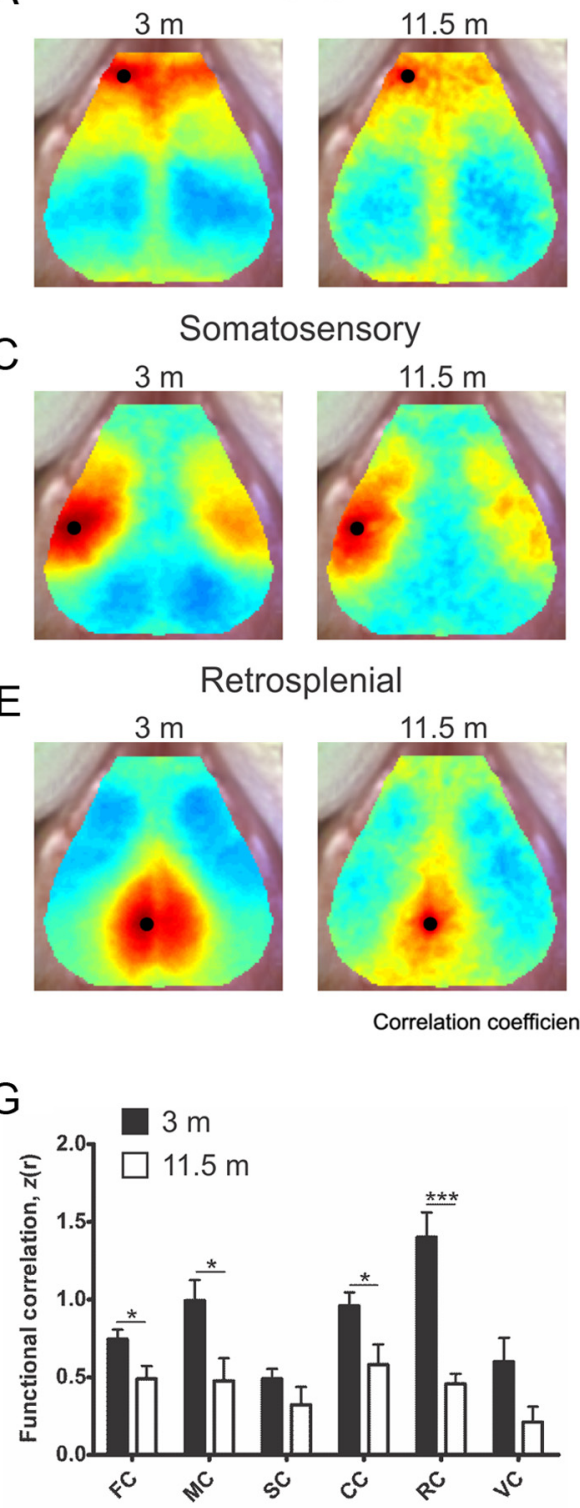

$11.5 \mathrm{~m}$

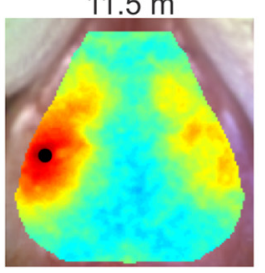

Retrosplenial

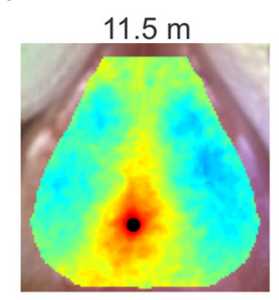

B

F

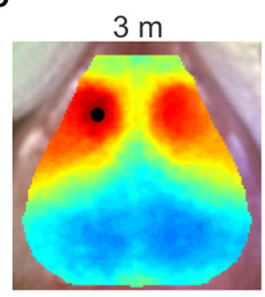

Motor
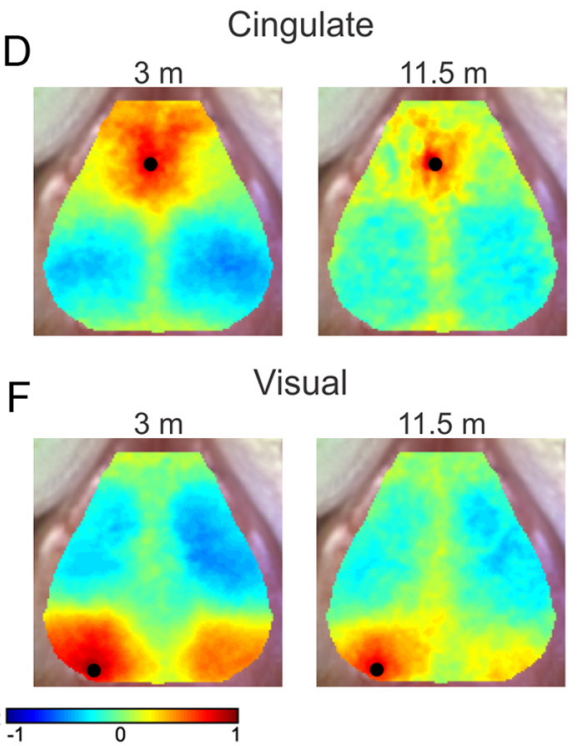

Visual

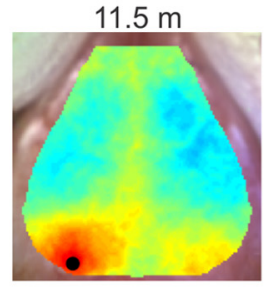

$\mathrm{H}$

Histological Atlas

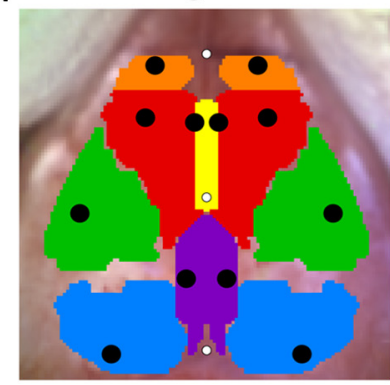

$11.5 \mathrm{~m}$

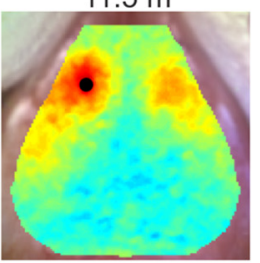

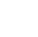


A

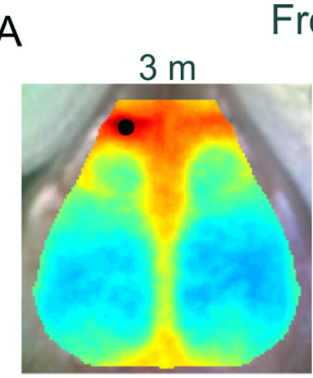

Frontal

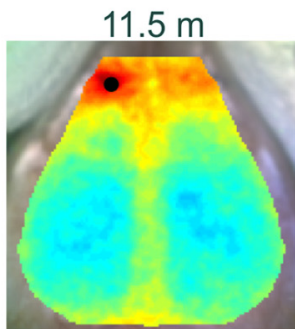

Somatosensory

C
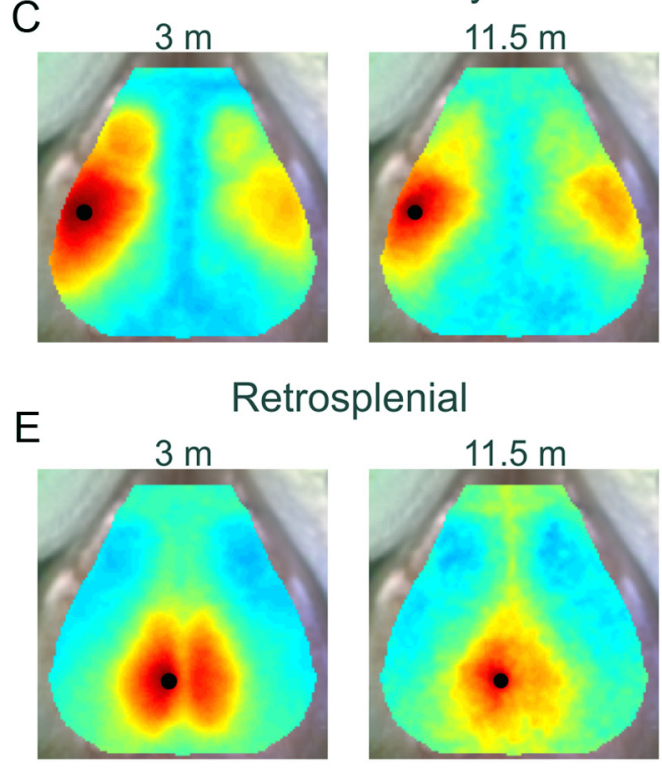

B

Motor

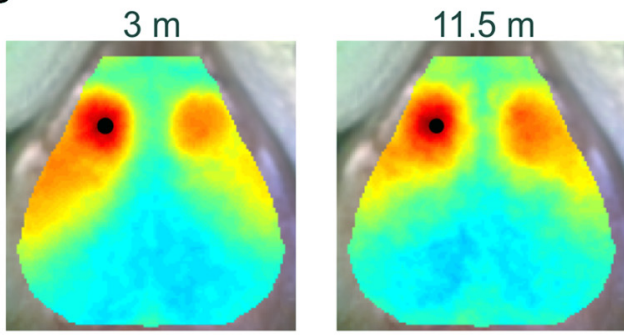

Cingulate

D
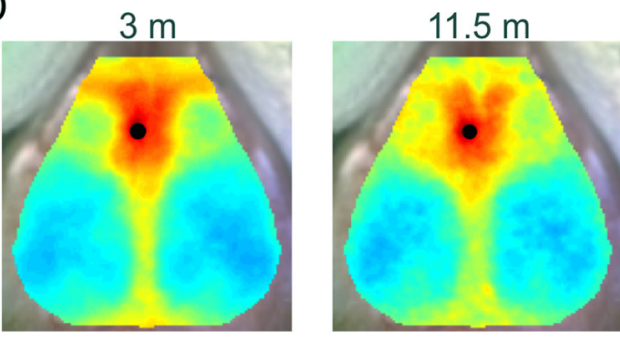

F

Visual
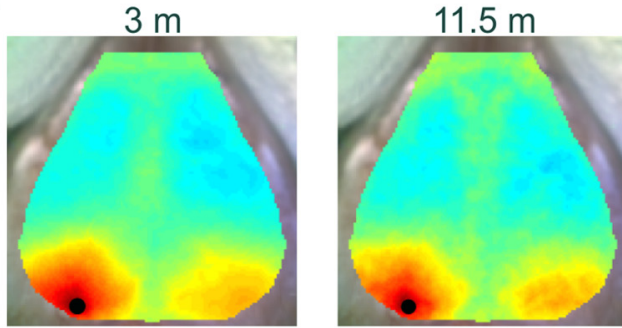

Correlation coefficient:

G

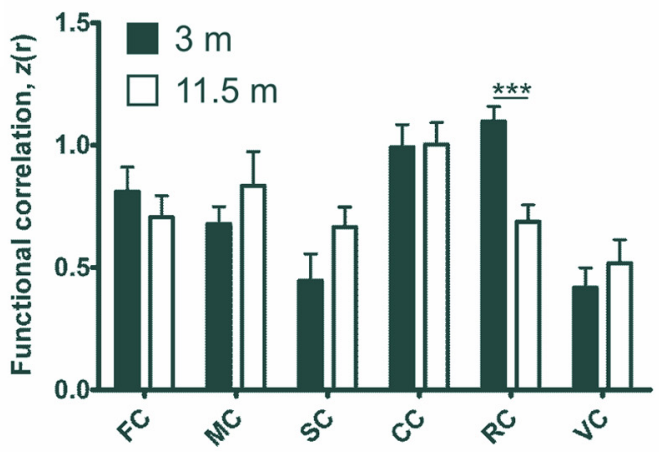

Figure 2. Normal aging is associated with decreased functional connectivity in retrosplenial cortex. $\boldsymbol{A}-\boldsymbol{F}$, Composite, group-averaged, functional correlation maps of frontal (FC, $\boldsymbol{A}$ ), motor (MC, $\boldsymbol{B})$, somatosensory $(\mathrm{SC}, \boldsymbol{C})$, cingulate $(C C, \boldsymbol{D})$, retrosplenial $(\mathrm{RC}, \boldsymbol{E})$ and visual $(\mathrm{VC}, \boldsymbol{F})$ cortices in young and older wild-type mice. Black circles denote seed position. $\boldsymbol{G}$, Regional bilateral functional correlation in young (black bars) and older (white bars) wild-type mice (young, $n=10$; older, $n=13$ ); ${ }^{* * *} p<0.001$. Values represent mean \pm SEM.

$30 \mathrm{~Hz}$. The field-of-view covered the majority of the convexity of the cerebral cortex $\left(\sim 1 \mathrm{~cm}^{2}\right)$.

Image processing. Data from all mice were subject to an initial quality check before spectroscopic analysis. Data runs $(5 \mathrm{~min})$ in which temporal variation in reflected light level intensity exceeded $1 \%$ for any wavelength were excluded from further analysis. This preliminary analysis resulted in 15-45 min of data per mouse. For each pixel, the differential light intensity at each wavelength was converted to differential absorption. The multi-wavelength absorption coefficient data were then converted to hemoglobin concentration changes for each pixel and time point. The subsequent $\mathrm{fc}$ analysis used the spatio-temporal $\Delta \mathrm{HbO}_{2}$ data (White et al., 2009).
Data were filtered to the functional connectivity band $(0.009-0.08 \mathrm{~Hz})$ following previous human fMRI algorithms (Fox et al., 2005), resampled from $30 \mathrm{~Hz}$ to $1 \mathrm{~Hz}$, and a brain mask was created containing all pixels labeled as brain. Time traces of all brain pixels were averaged to create a global brain signal. This global signal was regressed from each pixel's time trace to remove global sources of variance, as is common in human fMRI algorithms.

Atlas registration. All image sequences and brain masks were affinetransformed to a common atlas space (Franklin and Paxinos, 1996) determined by the coordinates of olfactory bulb and cerebrum, bregma, and lambda. The anterior-posterior stretch was set equal to the mediallateral stretch. To ensure all comparisons were performed only on the 
A

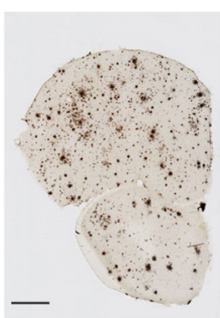

E

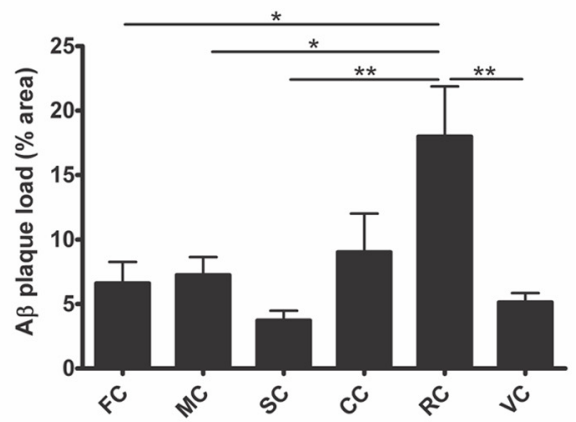

G

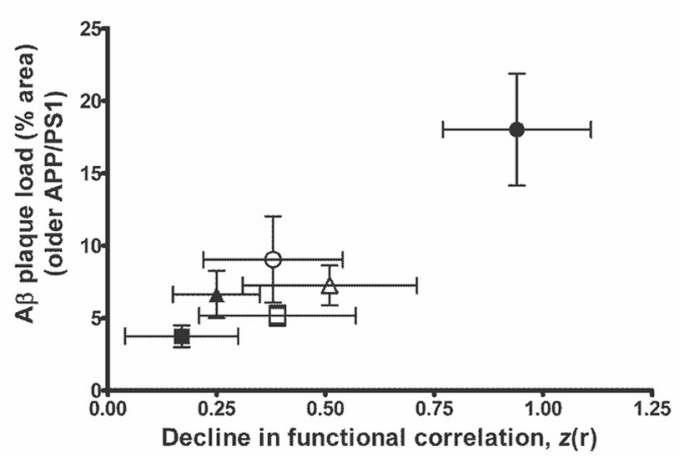

C

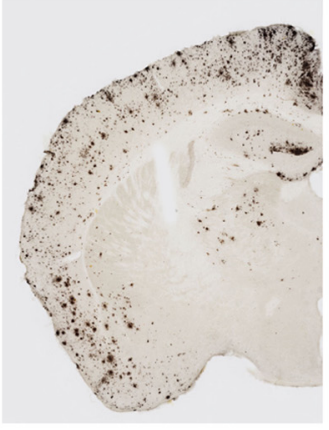

F

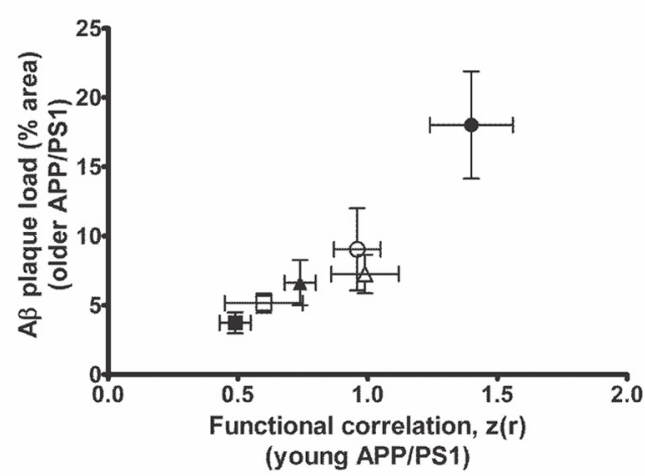

$\Delta$ Frontal

$\triangle$ Motor

Somatosensory

Cingulate

Retrosplenial

Visual

Figure 3. Regional bilateral functional connectivity in APP/PS1 mice is predictive of and affected by regional plaque deposition. $A-D$, Representative brain sections from older APP/PS1 mice stained with biotinylated HJ3.4 antibody (anti-A $\beta 1-13$ ) to visualize $A \beta$ immunopositive plaques ( $n=4 /$ group). Scale bar: (in $\boldsymbol{A}) \boldsymbol{A}-\boldsymbol{D}, 500 \mu \mathrm{m}$. $\boldsymbol{E}$, Percentage area occupied by $A \beta$ deposition in frontal (FC), motor (MC), somatosensory (SC), cingulate (CC), retrosplenial (RC), and visual (VC) cortices; ${ }^{*} p<0.05,{ }^{* *} p<0.01$. $F$, Regional bilateral correlation in young APP/PS1 mice plotted against A $\beta$ plaque deposition in each brain region of older APP/PS1 mice. G, Decline in bilateral correlation of older APP/PS1 mice plotted against A $\beta$ plaque deposition. Values represent mean \pm SEM.

brain area visualized in all mice, the intersection of each brain mask was calculated and made symmetric.

Seed-based functional connectivity measures. Seed placement was determined a priori using an anatomical atlas (Franklin and Paxinos, 1996). Twelve seed locations were positioned at coordinates corresponding to left and right frontal, motor, somatosensory, cingulate, retrosplenial, and visual cortices. Seed time traces were calculated by averaging time traces within $0.25 \mathrm{~mm}$ (30 pixels) of a seed locus and functional connectivity maps were created by correlating each seed time trace against those in every brain pixel. Individual functional connectivity maps were averaged within each cohort to generate consensus maps for each cohort. Regional bilateral correlations were determined by correlating the seed-based time trace in each functional area with that of its contralateral homolog, thus producing six bilateral correlation values for each mouse. Pearson $r$ values were converted to Fisher $Z$ measures using: $Z(r)=\operatorname{arctanh}(r)$ for all group comparisons. To further examine bilateral connectivity across the brain, every pixel in the brain was used as a seed center and its time trace was correlated with its contralateral homolog. Individual bilateral maps were averaged within each cohort to generate consensus bilateral connectivity maps.

$A \beta$ plaque deposition. Animals were transcardially perfused with chilled PBS, pH 7.4, with $0.3 \%$ heparin immediately following the final fcOIS session. Brains were removed and postfixed in $4 \%$ paraformaldehyde at $4^{\circ} \mathrm{C}$ overnight. Following fixation, brains were cryoprotected in $30 \%$ sucrose in PBS at $4^{\circ} \mathrm{C}$ for $72 \mathrm{~h}$. Using a freezing sliding microtome (Microm), serial coronal sections (50 $\mu \mathrm{m}$ thickness) were collected from frontal cortex to caudal hippocampus. Sections (separated by $300 \mu \mathrm{m}$ ) were stained with biotinylated HJ3.4 (anti-A $\beta 1-13$, mouse monoclonal antibody generated in-house) to visualize $\mathrm{A} \beta$ immunopositive plaques. Immunostained sections were scanned using a Nanozoomer slide scanner (Hamamatsu Photonics). Quantitative analysis of immunopositive staining was performed as described previously (Bero et al., 2011). Briefly, images of immunostained sections were exported with NDP viewer (Hamamatsu Photonics), converted to 8-bit grayscale using ACDSee Pro 2 software (ACD Systems), thresholded to highlight A $\beta$ - 
A

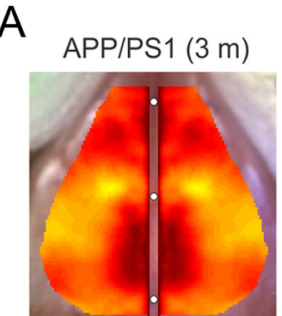

E

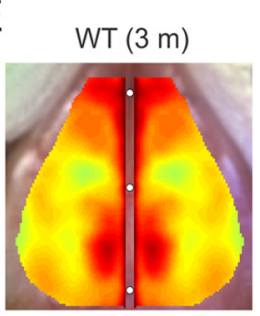

B

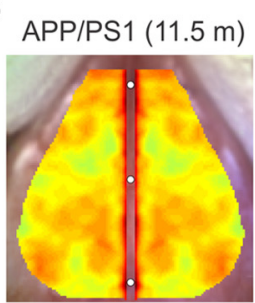

F
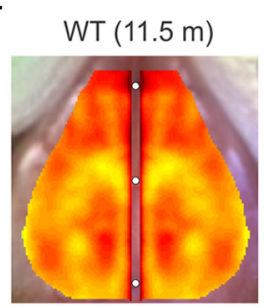

C

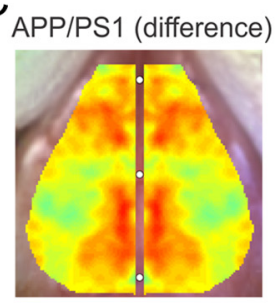

G

WT (difference)

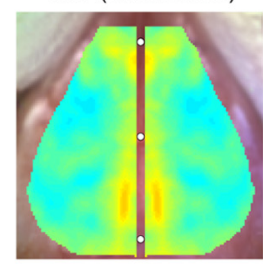

D p-value APP/PS1 Difference

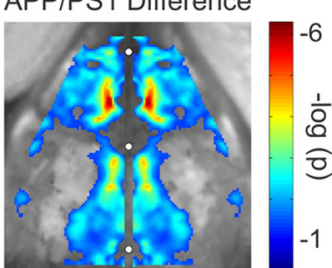

H p-value

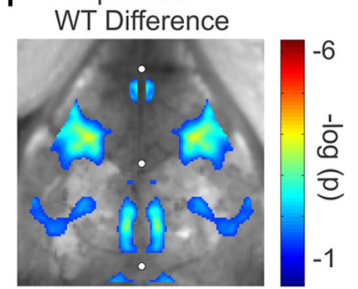

Fisher $z$ :

$-1.3$

0

1.3

Figure 4. $A \beta$ deposition is associated with degree of decline in local bilateral functional connectivity. $\boldsymbol{A}, \boldsymbol{B}$, Consensus bilateral functional connectivity maps generated for young $(\boldsymbol{A})$ and older $(\boldsymbol{B})$ APP/PS1 mice and young $(\boldsymbol{E})$ and older $(\boldsymbol{F})$ wild-type mice. $\boldsymbol{C}, \boldsymbol{G}$, Bilateral functional connectivity difference map between young and older APP/PS1 $(\boldsymbol{C})$ and wild-type $(\boldsymbol{G})$ mice. $\boldsymbol{D}, \boldsymbol{H}, p$ values of $\boldsymbol{C}$, $\mathbf{G}$, plotted on a $-\log _{10}$ scale. Note that in $\mathbf{C}, \mathbf{G}$, positive values represent age-related decline, whereas negative values represent age-related increase in bilateral functional connectivity. White circles denote anatomical landmarks for image coregistration.

specific staining and analyzed using ImageJ (NIH). A mouse brain atlas (Franklin and Paxinos, 1996) was used to identify frontal, motor, somatosensory, cingulate, retrosplenial, and visual cortices for quantitative analysis of $\mathrm{A} \beta$ immunopositive staining.

Statistical analysis. Statistical significance was determined by twotailed $t$ test if two groups were compared. When variance differed significantly between groups, Welch's $t$ test was used. One-way ANOVA followed by Tukey's post hoc test for multiple comparisons were used when more than two groups were compared. All statistical analyses were performed using Prism version 5.00 for Windows (GraphPad) or Matlab. Values were accepted as significant if $p<0.05$.

\section{Results}

A $\beta$ plaque deposition and normal aging are associated with functional connectivity disruption

To investigate the relationship between $\mathrm{A} \beta$ deposition and functional connectivity in a mouse model of cerebral $\beta$-amyloidosis, we used fcOIS imaging to examine bilateral functional connectivity magnitude in APP/PS1 mice before and following $\mathrm{A} \beta$ plaque deposition. This mouse model develops cerebral $\mathrm{A} \beta$ plaque deposition beginning at $4-5$ months of age (Jankowsky et al., 2004). Relative to young APP/PS1 mice, older APP/PS1 mice exhibited significant reductions in bilateral functional correlation strength in frontal $(Z(r), 3$ month, $0.74 \pm 0.06$; 11.5 month, $0.49 \pm 0.08 ; p=0.03)$, motor ( 3 month, $1.05 \pm 0.13 ; 11.5$ month, $0.48 \pm 0.15 ; p=0.022$ ), cingulate ( 3 month, $0.96 \pm 0.9 ; 11.5$ month, $0.58 \pm 0.13 ; p=0.031$ ), and retrosplenial ( 3 month, $1.4 \pm 0.16 ; 11.5$ month, $0.46 \pm 0.06 ; p=0.0009$ ) cortices. Significant differences were not observed in somatosensory ( 3 month, $0.49 \pm 0.06 ; 11.5$ month, $0.32 \pm 0.12 ; p=0.23)$ or visual $(3$ month, $0.6 \pm 0.15 ; 11.5$ month, $0.21 \pm 0.1 ; p=0.057)$ cortices (Fig. 1A-G; functional connectivity maps are population averages following affine transformation, $n=7$ (group).

We next sought to determine whether normal aging was associated with reduced bilateral functional connectivity in B6C3 wild-type mice. Compared with young wild-type mice, older wild-type mice exhibited a significant reduction in bilateral connectivity in retrosplenial cortex $(Z(r), 3$ month, $1.1 \pm 0.06 ; 11.5$ month, $0.69 \pm 0.07 ; p=0.0002$ ). In contrast, comparisons of bilateral functional connectivity between young and older wildtype mice were not significant in frontal ( 3 month, $0.81 \pm 0.1$; 11.5 month, $0.71 \pm 0.09 ; p=0.46$ ), motor ( 3 month, $0.68 \pm 0.07$; 11.5 month, $0.83 \pm 0.14 ; p=0.30$ ), somatosensory ( 3 month, $0.45 \pm 0.11 ; 11.5$ month, $0.67 \pm 0.08 ; p=0.14)$, cingulate $(3$ month, $0.99 \pm 0.09 ; 11.5$ month, $1.0 \pm 0.09 ; p=0.94)$ or visual $(3$ month, $0.42 \pm 0.08 ; 11.5$ month, $0.52 \pm 0.1 ; p=0.44)$ cortices [Fig. 2A-G; functional connectivity maps are population averages following affine transformation, $n=10$ (young) and 13 (older)]. These data suggest that bilateral functional connectivity magnitude is decreased during normal aging and is exacerbated by $\mathrm{A} \beta$ deposition.

\section{Bilateral functional connectivity magnitude predicts region- specific $\mathrm{A} \boldsymbol{\beta}$ plaque deposition}

To determine whether regional bilateral functional connectivity magnitude in young APP/PS1 mice before $\mathrm{A} \beta$ deposition was predictive of the regional vulnerability to $A \beta$ deposition in older APP/ PS1 mice, we characterized the distribution of $A \beta$ immunopositive plaque deposition in older APP/PS1 mice. Brain sections were immunostained with biotinylated $\mathrm{HJ} 3.4$ antibody, to $\mathrm{A} \beta$ amino acids $1-13$ (anti-A $\beta 1-13$ ), to determine the percentage area occupied by $\mathrm{A} \beta$ plaques in each region of interest (Fig. $3 A-D)$. We found that $\mathrm{A} \beta$ plaque deposition was greatest in retrosplenial cortex (18.01 \pm $3.86 \%)$, intermediate in cingulate $(9.04 \pm 2.97 \%)$, frontal $(6.63 \pm$ $1.63 \%)$, motor $(7.26 \pm 1.38 \%)$ cortices, and low in somatosensory $(3.74 \pm 0.75 \%)$ and visual $(5.16 \pm 0.69 \%)$ cortices (Fig. 3E; $n=4)$. Remarkably, we found that bilateral functional connectivity magnitude in young APP/PS1 mice before plaque deposition was proportional to the amount of $\mathrm{A} \beta$ plaque deposition in older APP/PS1 mice in a region-specific manner (Fig. $3 F$ ).

\section{Local $A \beta$ plaque deposition is associated with degree of local} functional connectivity decline

Quantitative analysis of regional $\mathrm{A} \beta$ deposition shows a strong association with regional functional connectivity decline (Fig. $3 G$ ). To more closely examine the spatial relationship between 
$\mathrm{A} \beta$ deposition and age-related decline in bilateral functional connectivity, fcOIS datasets from each mouse were affinetransformed to a common atlas space and individual bilateral maps were averaged within each cohort to generate a single, fullfield, consensus bilateral connectivity map for each cohort (Fig. $4 A, B, E, F)$. To examine pixel-wise, age-related changes in bilateral functional connectivity, consensus values generated from older mice were subtracted from consensus values generated from young mice of the same genotype (Fig. $4 C, G$ ) and compared (Fig. $4 D, H$ ). The resultant difference maps reveal local differences in the level of age-related functional decline in APP/PS1 and wild-type mice, and notably, reproduce the regional differences in bilateral functional connectivity reported above. These data further suggest that functional connectivity in mouse brain is reduced in normal aging and is exacerbated by $\mathrm{A} \beta$ deposition in a region-specific manner.

\section{Discussion}

Intrinsic resting-state functional connectivity represents highly organized synaptic activity within the brain (Pizoli et al., 2011). A close relationship between changes in hemoglobin oxygenation (i.e., resting fMRI and OIS imaging) and slow $(<0.1 \mathrm{~Hz})$ fluctuations in local field potentials has been established in human ( $\mathrm{He}$ et al., 2008) and rodent brain (Hahn et al., 2006; Lu et al., 2007). Disruption of functional connectivity is an important biomarker of neurological disease (Zhang and Raichle, 2010) and one that will likely yield new insights into pathophysiology and potentially reveal novel therapeutic avenues. However, the relationship between functional connectivity, $A \beta$ deposition, and normal aging in mouse brain remains unknown. Here, we used a novel fcOIS imaging approach (White et al., 2011) to characterize the relationship between functional connectivity, $\mathrm{A} \beta$ deposition, and normal aging in mouse brain. As expected, both positive (red) and negative (blue) correlations are present in the functional connectivity maps of Figure $1 A-F$ and Figure $2 A-F$, a finding consistent with our previous work in humans (Fox et al., 2005) and mice (White et al., 2011). Interpretation of anticorrelations warrants some caution, however, as global signal regression enforces the presence of anticorrelated pixels. As the present data suggest that fcOIS anticorrelations in mice are not robustly affected by aging or $A \beta$ deposition, we focused our analysis on alterations in the positive correlation. In accord with human data (Greicius et al., 2004; Hedden et al., 2009; Sheline et al., 2010; Drzezga et al., 2011; Jones et al., 2011; Mormino et al., 2011), we found that region-specific $A \beta$ accumulation is associated with significant reductions in bilateral functional connectivity magnitude in multiple brain systems of older APP/PS1 mice. Moreover, brain regions in which amyloid deposition was most prominent exhibited greater age-related declines in functional connectivity relative to areas of lesser plaque deposition. As $\mathrm{A} \beta$ plaque deposition is associated with local neuritic dystrophy and impaired synaptic integration in APP transgenic mice (Stern et al., 2004; Meyer-Luehmann et al., 2008) future application of fcOIS will permit examination of the molecular mechanisms that underlie the relationship between $A \beta$ deposition and impaired systemslevel functional connectivity.

In humans, normal age-related memory deficits are associated with decreased DMN functional correlation (Andrews-Hanna et al., 2007). Here, we found that retrosplenial cortex exhibits decreased bilateral functional connectivity in wild-type mice at 11.5 months of age. As age-related memory deficits are not present in wild-type mice at this age, and given that retrosplenial cortex is important for learning and memory (Corcoran et al., 2011), im- paired bilateral functional connectivity in retrosplenial cortex may represent an early event in age-related neural dysfunction. In this context, fcOIS may serve as a useful tool to investigate the molecular mechanisms that contribute to decreased functional connectivity and memory dysfunction associated with normal aging.

Intriguingly, we found that the magnitude of region-specific bilateral functional correlation in young APP/PS1 mice before the onset of $\mathrm{A} \beta$ plaque formation was proportional to the density of regional plaque deposition in older APP/PS1 mice. This finding is consistent with human data suggesting that areas that comprise the DMN exhibit disproportionately high intrinsic structural and functional connectivity in cognitively normal young adults and are among the brain regions most vulnerable to $\mathrm{A} \beta$ deposition in $\mathrm{AD}$ (Hagmann et al., 2008; Buckner et al., 2009). Our laboratory has recently demonstrated that regional differences in endogenous neuronal activity are closely associated with interstitial fluid $A \beta$ levels and are a key determinant of region-specific $\mathrm{A} \beta$ plaque deposition in APP transgenic mice (Bero et al., 2011). As the present data suggest that regional bilateral functional connectivity magnitude in young mice before plaque deposition is also associated with the level of subsequent $\mathrm{A} \beta$ plaque deposition, it is likely that similar associations between neural activity/metabolism, functional connectivity, and susceptibility to amyloid deposition exist in mouse brain. However, such convergence has not yet been directly examined to determine how these mechanisms may independently or coordinately regulate $\mathrm{A} \beta$ metabolism.

The results of the current study describing age and pathologyrelated decreases in functional connectivity are consistent with changes previously observed in human brain. In human brain however, normal aging and $\mathrm{AD}$ are also associated with regionspecific increases in functional connectivity (Jones et al., 2011; Mormino et al., 2011). Although we did observe local increases in functional connectivity near the somato-motor seeds of wildtype mice, human data suggest that prefrontal cortex harbors the most significant increase in anterior DMN functional connectivity in $\mathrm{AD}$ (Jones et al., 2011). As brain areas that comprise mouse prefrontal cortex reside outside the field-of-view of the current OIS system, it remains possible that age or pathology-related increases in functional connectivity are present in more ventrally located regions. In future studies, these deeper brain regions might be imaged using diffuse optical tomography (White et al., 2009).

A major challenge facing the neuroscience community is the paucity of clinically translatable assays that examine neural network function and integrity. Given that resting-state functional connectivity measures have provided valuable insight into the functional organization of the human brain and have yielded examples of how such organization is disrupted in disease, use of fcOIS in the setting of genetic and pharmacological manipulations provides an opportunity to elucidate the role of specific genes/proteins in systems-level functional connectivity in healthy brain and its disruption in mouse models of neurological and neurodegenerative disorders. The clinically translatable nature of fcOIS also positions this technique as a useful tool with which to examine the efficacy of pharmacological or behavioral interventions aimed at restoring neural network integrity.

\section{References}

Andrews-Hanna JR, Snyder AZ, Vincent JL, Lustig C, Head D, Raichle ME, Buckner RL (2007) Disruption of large-scale brain systems in advanced aging. Neuron 56:924-935. 
Bero AW, Yan P, Roh JH, Cirrito JR, Stewart FR, Raichle ME, Lee JM, Holtzman DM (2011) Neuronal activity regulates the regional vulnerability to amyloid-beta deposition. Nat Neurosci 14:750-756.

Buckner RL, Snyder AZ, Shannon BJ, LaRossa G, Sachs R, Fotenos AF, Sheline YI, Klunk WE, Mathis CA, Morris JC, Mintun MA (2005) Molecular, structural, and functional characterization of Alzheimer's disease: evidence for a relationship between default activity, amyloid, and memory. J Neurosci 25:7709-7717.

Buckner RL, Sepulcre J, Talukdar T, Krienen FM, Liu H, Hedden T, AndrewsHanna JR, Sperling RA, Johnson KA (2009) Cortical hubs revealed by intrinsic functional connectivity: mapping, assessment of stability, and relation to Alzheimer's disease. J Neurosci 29:1860-1873.

Corcoran KA, Donnan MD, Tronson NC, Guzmán YF, Gao C, Jovasevic V, Guedea AL, Radulovic J (2011) NMDA receptors in retrosplenial cortex are necessary for retrieval of recent and remote context fear memory. J Neurosci 31:11655-11659.

Drzezga A, Becker JA, Van Dijk KR, Sreenivasan A, Talukdar T, Sullivan C, Schultz AP, Sepulcre J, Putcha D, Greve D, Johnson KA, Sperling RA (2011) Neuronal dysfunction and disconnection of cortical hubs in nondemented subjects with elevated amyloid burden. Brain 134:1635-1646.

Fox MD, Snyder AZ, Vincent JL, Corbetta M, Van Essen DC, Raichle ME (2005) The human brain is intrinsically organized into dynamic, anticorrelated functional networks. Proc Natl Acad Sci U S A 102:9673-9678.

Franklin KB, Paxinos G (1996) The mouse brain in stereotaxic coordinates, Ed 1. San Diego: Academic.

Greicius MD, Srivastava G, Reiss AL, Menon V (2004) Default-mode network activity distinguishes Alzheimer's disease from healthy aging: evidence from functional MRI. Proc Natl Acad Sci U S A 101:4637-4642.

Hagmann P, Cammoun L, Gigandet X, Meuli R, Honey CJ, Wedeen VJ, Sporns O (2008) Mapping the structural core of human cerebral cortex. PLoS Biol 6:e159.

Hahn TT, Sakmann B, Mehta MR (2006) Phase-locking of hippocampal interneurons' membrane potential to neocortical up-down states. Nat Neurosci 9:1359-1361.

He BJ, Snyder AZ, Zempel JM, Smyth MD, Raichle ME (2008) Electrophysiological correlates of the brain's intrinsic large-scale functional architecture. Proc Natl Acad Sci U S A 105:16039-16044.

Hedden T, Van Dijk KR, Becker JA, Mehta A, Sperling RA, Johnson KA, Buckner RL (2009) Disruption of functional connectivity in clinically normal older adults harboring amyloid burden. J Neurosci 29:12686-12694.

Jankowsky JL, Fadale DJ, Anderson J, Xu GM, Gonzales V, Jenkins NA, Copeland NG, Lee MK, Younkin LH, Wagner SL, Younkin SG, Borchelt DR (2004) Mutant presenilins specifically elevate the levels of the 42 residue beta-amyloid peptide in vivo: evidence for augmentation of a 42-specific gamma secretase. Hum Mol Genet 13:159-170.

Jones DT, Machulda MM, Vemuri P, McDade EM, Zeng G, Senjem ML,
Gunter JL, Przybelski SA, Avula RT, Knopman DS, Boeve BF, Petersen RC, Jack CR Jr (2011) Age-related changes in the default mode network are more advanced in Alzheimer disease. Neurology 77:1524-1531.

Kuchibhotla KV, Goldman ST, Lattarulo CR, Wu HY, Hyman BT, Bacskai BJ (2008) Abeta plaques lead to aberrant regulation of calcium homeostasis in vivo resulting in structural and functional disruption of neuronal networks. Neuron 59:214-225.

Lu H, Zuo Y, Gu H, Waltz JA, Zhan W, Scholl CA, Rea W, Yang Y, Stein EA (2007) Synchronized delta oscillations correlate with the resting-state function MRI signal. Proc Natl Acad Sci U S A 104:18265-18269.

Meyer-Luehmann M, Spires-Jones TL, Prada C, Garcia-Alloza M, de Calignon A, Rozkalne A, Koenigsknecht-Talboo J, Holtzman DM, Bacskai BJ, Hyman BT (2008) Rapid appearance and local toxicity of amyloid-beta plaques in a mouse model of Alzheimer's disease. Nature 451:720-724.

Mormino EC, Smiljic A, Hayenga AO, Onami SH, Greicius MD, Rabinovici GD, Janabi M, Baker SL, Yen IV, Madison CM, Miller BL, Jagust WJ (2011) Relationships between beta-amyloid and functional connectivity in different components of the default mode network in aging. Cereb Cortex 21:2399-2407.

Pizoli CE, Shah MN, Snyder AZ, Shimony JS, Limbrick DD, Raichle ME, Schlaggar BL, Smyth MD (2011) Resting-state activity in development and maintenance of normal brain function. Proc Natl Acad Sci U S A 108:11638-11643.

Selkoe DJ (2001) Alzheimer's disease: genes, proteins, and therapy. Physiol Rev 81:741-766.

Sheline YI, Raichle ME, Snyder AZ, Morris JC, Head D, Wang S, Mintun MA (2010) Amyloid plaques disrupt resting state default mode network connectivity in cognitively normal elderly. Biol Psychiatry 67:584-587.

Stern EA, Bacskai BJ, Hickey GA, Attenello FJ, Lombardo JA, Hyman BT (2004) Cortical synaptic integration in vivo is disrupted by amyloid-beta plaques. J Neurosci 24:4535-4540.

Vlassenko AG, Vaishnavi SN, Couture L, Sacco D, Shannon BJ, Mach RH, Morris JC, Raichle ME, Mintun MA (2010) Spatial correlation between brain aerobic glycolysis and amyloid-beta (Abeta) deposition. Proc Natl Acad Sci U S A 107:17763-17767.

White BR, Snyder AZ, Cohen AL, Petersen SE, Raichle ME, Schlaggar BL, Culver JP (2009) Resting-state functional connectivity in the human brain revealed with diffuse optical tomography. Neuroimage 47:148-156.

White BR, Bauer AQ, Snyder AZ, Schlaggar BL, Lee JM, Culver JP (2011) Imaging of functional connectivity in the mouse brain. PLoS One 6:e16322.

Yan P, Bero AW, Cirrito JR, Xiao Q, Hu X, Wang Y, Gonzales E, Holtzman DM, Lee JM (2009) Characterizing the appearance and growth of amyloid plaques in APP/PS1 mice. J Neurosci 29:10706-10714.

Zhang D, Raichle ME (2010) Disease and the brain's dark energy. Nat Rev Neurol 6:15-28. 